\title{
CFD Simulation of Flow Field for Pilot Operated Check Valve
}

\author{
Liqiang Zhang ${ }^{1,2}$, Wenjing Zheng ${ }^{1}$, Jingfang Zhang ${ }^{1}$, Min Lian ${ }^{1}$ \\ ${ }^{1}$ School of Energy and Power Engineering, Lanzhou University of Technology, Lanzhou 730050, \\ China \\ ${ }^{2}$ Wenzhou Academy of Pump and Valve Engineering, Lanzhou University of Technology, Wenzhou \\ 325105, China
}

Keywords: pilot operated check; valve flow field; cavitation numerical simulation; CFD.

\begin{abstract}
By computational fluid dynamics (CFD) technology, the numerical simulation of internal flow field for pilot operated check valve is determined. In the paper, by building flow channel model under different valve opening and changing its import and export pressure, it gets the pressure distribution contour and velocity vector distribution. Analyzing the result, it is found that the corner valve port of low pressure is serious and flow field reach maximum speed. It is easy to produce cavitation, noise and shock. Improved the flow structure and compared cavitation index curve, it is found the cavitation index increases, which can reduce the possibility of cavitation.
\end{abstract}

\section{Introduction}

Pilot operated check valve is a reverse opening controllable valve, which is widely used in metallurgical hydraulic system and cylinder for clamping and supporting ${ }^{[1]}$.Cavitation is one of the most common phenomenon in hydraulic components, which directly affects the stability of the hydraulic system ${ }^{[2]}$. Due to high differential pressure between entry and exit, cavitation is easily produced, vibration and noise. Simulating its flow field to identify the main factors affecting the hydraulic control valve performance has important significance to improve the channel design ${ }^{[3]}$.

\section{Working principle of pilot operated check valve}

Structure of YD type pilot operated check valve is shown in figure 1, which is composed with piston, valve core, spring, valve body and pipe plug. When the port K doesn't have pressure oil, it equivalents to an ordinary check valve whose pressure oil only can flow from P1 to P2. When there has controlled pressure in port $\mathrm{K}$, the oil flow from P2 to P1 realizing the reverse flow.

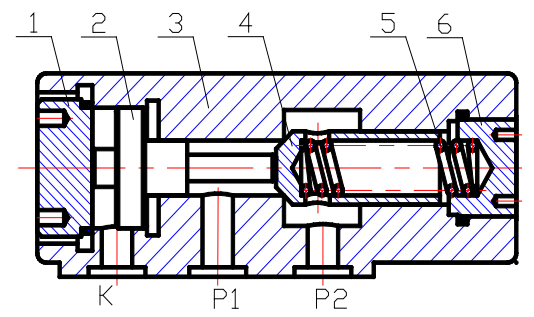

Fig.1 Structure of YD type pilot operated check valve 1,6-pipe plug 2-piston 3-valve body 4-valve core 5- spring

\section{CFD Computing Model}

The basic equations of fluid. When the fluid flow is turbulent, numerically computed is based on the basic equations of fluid and additional turbulent transport equations to calculate the flow field.

Mass conservation equation

$$
\frac{\partial \rho u_{i}}{\partial x_{i}}=0
$$

In the formula: $\rho$ - fluid density; $u_{i}$-the velocity of direction $i$.

Momentum conservation equation 


$$
\frac{\partial}{\partial t}\left(\rho u_{i}\right)+\frac{\partial}{\partial x_{j}}\left(\rho u_{i} u_{j}\right)=-\frac{\partial p}{\partial x_{j}}+\frac{\partial \tau_{i j}}{\partial x_{j}}+\rho g_{i}+F_{i}
$$

In the formula: $u_{j}$ - speed of direction $j ; \tau_{i j}$-stress tensor; $\rho g_{i}$-gravitational force volume.

Energy conservation equation ${ }^{[4]}$

$$
\frac{\partial}{\partial t}(\rho E)+\frac{\partial}{\partial x_{i}}\left[u_{i}(\rho E+p)\right]=\frac{\partial}{\partial x_{i}}\left(k_{e f f} \frac{\partial T}{\partial x_{i}}\right)-\sum h_{j^{\prime}} J_{j^{\prime}}+u_{j}\left(\tau_{i j}\right)_{\text {eff }}+S_{h}
$$

In formula: $k_{\text {eff }}$ - effective thermal conductivity; $J_{j^{\prime}}$-diffusion flux of components $j^{\prime}$;

$S_{h}$-source items of chemical reaction heat and other volume heat sources ${ }^{[5]}$.

Geometric modeling and grid division. Ignoring the asymmetry of flow channel of pilot operated check valve and simplifing it as a two-dimensional axis symmetric flow field build the channel structures of different valve throttle opening ${ }^{[6]}$. Then the model is imported into Gambit to generate grid in figure 2. The mesh density is bigger to obtain high accuracy of simulation. With complex and changeable throttle fluid flow, grid refinement is performed on it to accurately reflect the flow near the valve port, as shown in figure 3.

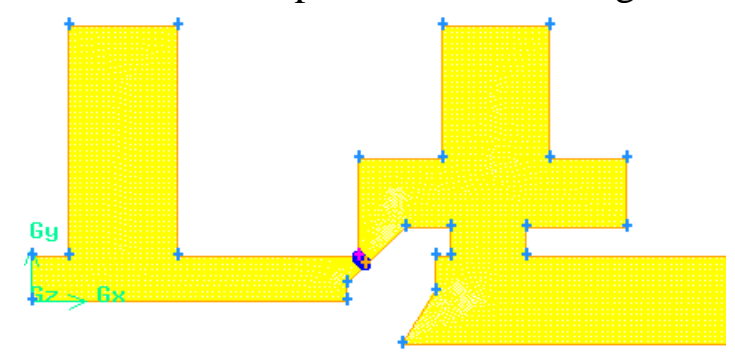

Fig. 2 Grid model

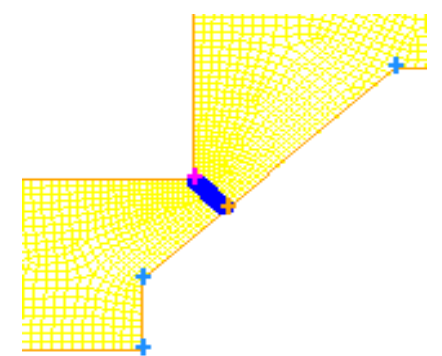

Fig. 3 Grid refinement of valve port

Calculation condition. It can make following assumptions in the numerical simulation process. Flow media is ordinary hydraulic oil, effect of gravity doesn't be considered ${ }^{[7]}$.The density is $889 \mathrm{~kg} / \mathrm{m}^{3}$, dynamic viscosity is $0.00103 \mathrm{~Pa} / \mathrm{s}$. Because Reynolds is greater than 2000, using standard $\kappa \sim \varepsilon$ turbulent model and SIMPLE method. The inlet and outlet boundary condition are both pressure. The outlet pressure is $5 \mathrm{MPa}$, and the inlet pressure are $15 \mathrm{MPa}, 20 \mathrm{MPa}, 25 \mathrm{MPa}$, 30MPa, $32 \mathrm{MPa}$.

\section{Simulation Analysis}

Simulation results of fixed opening. As shown in Figure 4, the pressure indicates gradient descent. There is a certain amount of pressure losses at the orifice, and large pressure gradient. This is because velocity increases when the constant fluid flow through throttle. At the same time, the negative pressure is generated near the region of throttle, and the lowest pressure reaches $-2.17 \mathrm{MPa}$.

The velocity vector is shown in figure 5 . Because of mutations of the flow area, the flow velocity increases to $197 \mathrm{~m} / \mathrm{s}$ after the orifice, and the maximum speed of region is distributed in the whole orifice, where the mainstream will be separated from the wall.

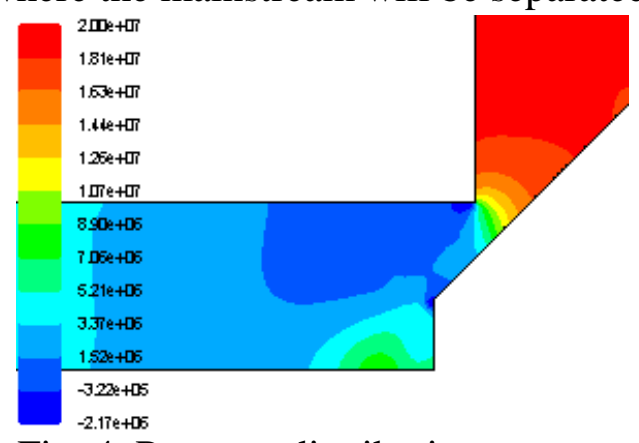

Fig. 4 Pressure distribution contours

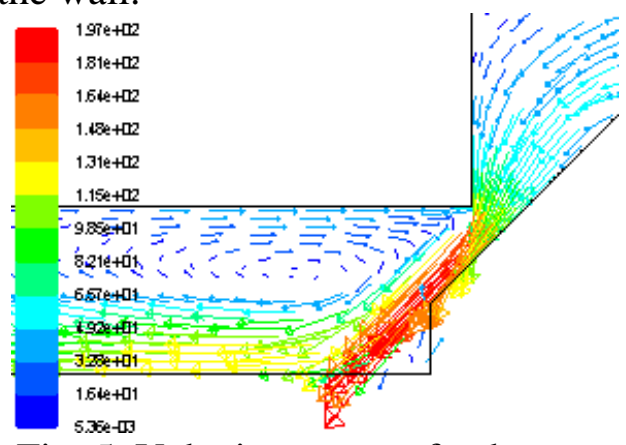

Fig. 5 Velocity vector of valve port

Simulation results of different opening and different differential pressure. It can be seen from figure 6 that the bigger valve opening is, the lower outlet pressure is. With the differential pressure 
becoming higher, the trend of pressure dropping is greater. When the opening is fixed, it is more obvious of negative pressure with the greater differential pressure. When opening is $2 \mathrm{~mm}$ and differential pressure is $27 \mathrm{MPa}$, the minimum pressure reaches to $-13.1 \mathrm{MPa}$.

It can be seen from figure 7 that the maximum speed of the valve port presents an increased trend as the inlet and outlet differential pressure and the opening increasing. When the opening is $2 \mathrm{~mm}$ and differential pressure is $27 \mathrm{MP}$, the maximum speed is up to $271 \mathrm{~m} / \mathrm{s}$.

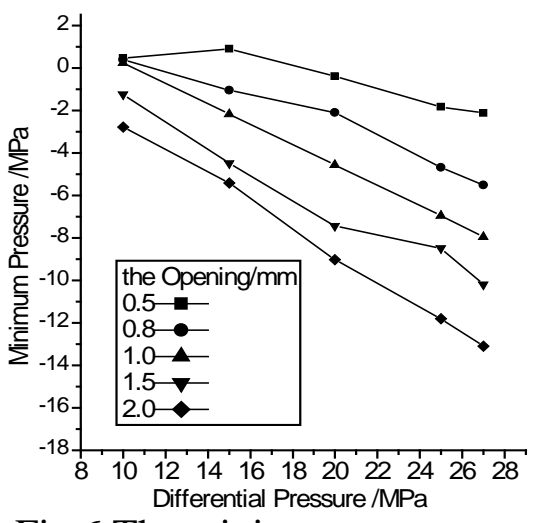

Fig. 6 The minimum pressure curve

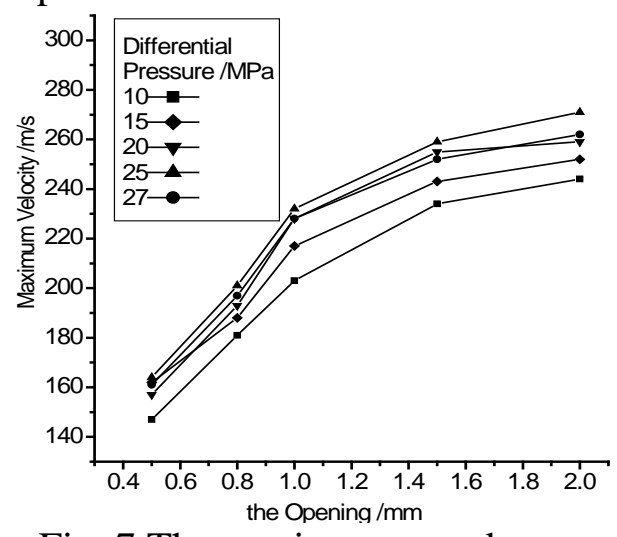

Fig. 7 The maximum speed curve

\section{Structure optimization of the pilot operated check valve}

The optimized Structure. In order to make pressure dropping reduce changing the structure of the valve body into two-step throttle. The improved structure is shown in figure 8 .

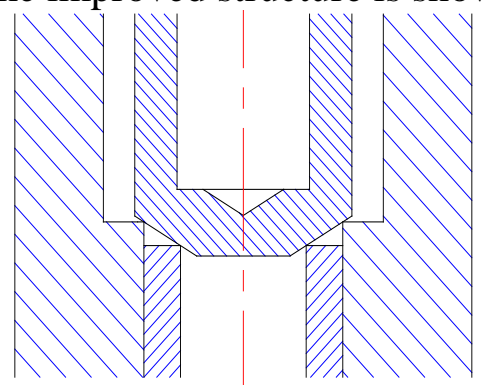

Fig. 8 The optimized structure

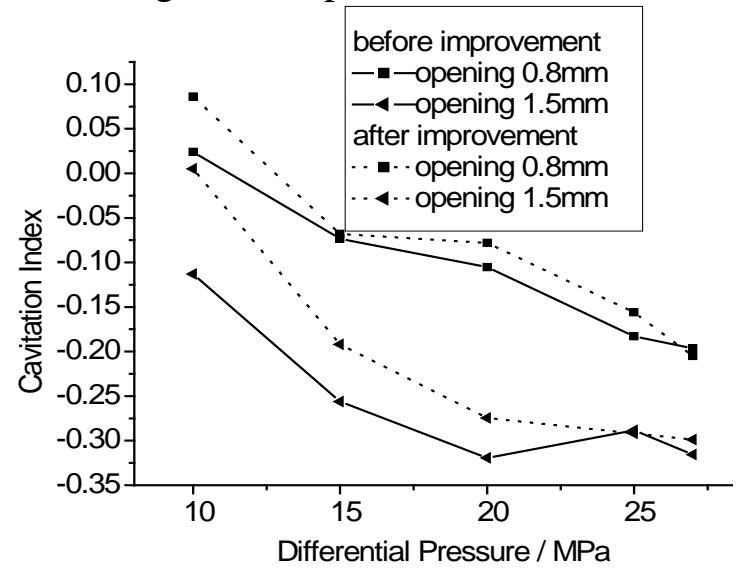

Fig. 9 Cavitation index curve of before and after improvemen

Cavitation analysis of optimized Structure. When the pressure is lower than the pressure of air and oil separation, the gas dissolved in oil will come out forming small bubble flowing with the fluid, which is called the phenomenon of cavitation ${ }^{[8]}$. It will not only reduce the component life and working efficiency, but also generate noise and vibration.

Cavitation can be reduced or prevented by controlling the oil pressure is higher than the air separation pressure at certain temperature ${ }^{[9]}$. It can use the cavitation index to measure the possibility of cavitation, defined as 


$$
\sigma=\frac{P-P_{v}}{\frac{1}{2} \rho v^{2}}
$$

In the formula: $P$ - absolute pressure (pa); $P_{v}$-the gasification pressure (pa); $v$-velocity at the moment $(\mathrm{m} / \mathrm{s}) ; \rho$ - fluid density $\left(\mathrm{kg} / \mathrm{m}^{3}\right)$.

As shown in figure 9, the cavitation index near the valve port are negative basically. The greater value of $\sigma$ is, the much little possibility of the occurrence of cavitation is, which means anti-cavitation performance is better. When differential pressure is $27 \mathrm{MPa}$, cavitation index reaches -0.316 before improvement which reflects the cavitation phenomenon is serious; with the structure improved by two-step throttle, cavitation index increases to -0.258 , which makes the cavitation decrease. Moreover, the index increases more obviously by changing structure under the situation of large opening.

\section{Conclusions}

At the throttle, velocity increases rapidly and pressure decreases because of the flow area decreased suddenly. The bigger valve opening and the differential pressure are, the higher the maximum velocity at the orifice is, and the smaller the lowest pressure is.

In the region of negative pressure, cavitation is easy to produce. With increasing of the opening and differential pressure, cavitation is more serious. Designed two-step throttle structure to optimize the throttle of pilot operated check valve, the inlet and outlet pressure is reduced, and cavitation index is increased obviously which makes the cavitation phenomenon decreased.

\section{References}

[1]Dexin Zhao, Ruibo Zhao. Analysis and Optimize on a Pilot Operated Check Valve[J]. Mechanical Manufacturing, 2011, 49 (560): p.14-16.

[2]Jianfeng Zhi. Cavitation and Countermeasures of Engineering Machinery Hydraulic Valve [J]. Machine Tool\&Hydraulics, 2008, 36(7): p.6-7.

[3]Zhikai He. Simulation Analysis of Prop Pilot Operated Check Valve and Research on Visualization of Flow Field [D]. Taiyuan: Taiyuan University and Technology, 2012: p.60-65.

[4]Hui Li, Jian Ke, Xiaohong Liu. CFD Analysis of the Structure Characteristics for the Hydraulic Poppet Valve[J]. Fluid Machine,2009,37(9): p.33-36.

[5]Wen Hua, Chenbo Yin. Numerical Simulation and Experimental Study on Cavitation of Valve Port [J]. Hydraulics \& Pneumatics,2010(4): p.14-16.

[6]Jingtao Wang, Zhangyong Wu, Xiaobo Wang. Flow Field Analysis and Structural Optimization of Hydraulic Poppet Valve Based on ANSYS [J]. Machine Tool\&Hydraulics, 2011, 39(13): p. 128-130.

[7]Hong Gao, Xin Fu. Investigation into Cavitation with in Holding Valve [J]. Hydraulics \& Pneumatics,2002(7): p.22-23.

[8]Wei Xie, Hua Zhou, Yongjun Gong. Research for Testing System of Cavitation Within Water Hydraulic Valve[J]. Hydraulics \&Pneumatics, 2004(5): p.14-17.

[9]Dalin Yue, Zisheng Lian. Numerical Simulation and Visual Analysis of Flow Field of Hydraulic Operated Check Valve in Hydraulic Powered Support [J]. Coal Mine Machinery, 2008, 29(5): p. 40-42. 